\title{
Experimental Studies on Micropumps Using Rotational/Reciprocating Motions of Magnetic Material Balls
}

\author{
Hiroshige Kumamaru*, Yoshio Nomura, Fuma Sakata, Hayata Fujiwara, Kazuhiro Itoh
}

Department of Mechanical Engineering, Graduate School of Engineering, University of Hyogo, Himeji, Japan

Email address:

kumamaru@eng.u-hyogo.ac.jp (H. Kumamaru)

*Corresponding author

\section{To cite this article:}

Hiroshige Kumamaru, Yoshio Nomura, Fuma Sakata, Hayata Fujiwara, Kazuhiro Itoh. Experimental Studies on Micropumps Using Rotational/Reciprocating Motions of Magnetic Material Balls. International Journal of Mechanical Engineering and Applications. Vol. 5, No. 5, 2017, pp. 247-252. doi: 10.11648/j.ijmea.20170505.12

Received: July 10, 2017; Accepted: July 21, 2017; Published: September 18, 2017

\begin{abstract}
In application of micropumps to new fields in chemistry, biology, medical science and others, smaller sizes are supposed to be important rather than higher pump performance. In this study, considering from such a view point, micropumps using rotational and reciprocating motions of magnetic material balls were proposed and studied experimentally. The pump performance, i.e. the relation between flow rate and pump head are measured from liquid level changes in two containers connected to the inlet and outlet of the micropump. For the rotational motion micropump, while the maximum flow rate obtained, $\sim 2 \mathrm{~mL} / \mathrm{min}$, is large enough as a micropump, the maximum pump head achieved, $\sim 15 \mathrm{~mm}$, is small even for a micropump. It is desirable to increase the pump head furthermore for this micropump. For the reciprocating motion micropump, the maximum flow rate obtained and the maximum pump head achieved are $\sim 7.5 \mathrm{~mL} / \mathrm{min}$ and $\sim 625 \mathrm{~mm}$, respectively. These values of the pump performance are sufficient as a micropump. Both the micropumps can be incorporated into microfluidic devices (tips) and can pump arbitrary kind of liquid.
\end{abstract}

Keywords: Micropump, Magnetic Material Ball, Rotational Motion, Reciprocating Motion, Pump Performance

\section{Introduction}

From the 1980's, various kinds of microfluidic devices (tips) have been developed for a wide range of application, from chemical analysis systems to actuating systems in medical science and biology. Many researchers have tried to develop micropumps to be applied to microfluidic devices based on various principles and methods. Recent reviews on micropumps are available in [1-5]. Usually, micropumps can be classified into two groups, i.e. mechanical/displacement types and non-mechanical/dynamic types [2-4, 6]. A diaphragm micropump with check valves and an electroosmotic micropump using porous electroosmotic material, belonging to the former and latter types, respectively, are almost at the stage of practical application, particularly from the viewpoint of pump performance (i.e. the maximum flow rate and the maximum pump head) $[6,7]$. However, both the diaphragm and electroosmotic micropumps cannot be incorporated into microfluidic devices (tips), while the electroosmotic micropump can be loaded on (attached to) a microfluidic device (tip). Also, the electroosmotic micropump can pump only electrolyte solution.

In parallel, microfluidic devices (tips) have been considered to be applied to new fields in chemistry, biology, medical science and others. In application of micropumps to such fields, smaller sizes are supposed to be important rather than higher pump performance [1-5]. Considering from such a view point, micropumps that can be incorporated into microfluidic devices and can pump arbitrary kind of liquid have been developed in this study.

First, in this study, is tested a micropump using rotational motion of magnetic material balls, based on basically the same principle as for Hatch et al.'s pump [8], explained in the next chapter. However, magnetic fluid in Hatch et al.'s micropump is replaced by magnetic material balls in the 
present pump. Secondly, a micropump using reciprocating motion of magnetic material balls is developed in this study. Both the present micropumps can be incorporated into microfluidic devices (tips) and can pump arbitrary kind of liquid.

\section{Micropump Using Magnetic Fluid or Magnetic Material Ball}

Several researchers have made efforts to carry out experiments for micropumps using magnetic fluid or magnetic material balls. Hatch et al. proposed a micropump using rotational motion of magnetic fluid shown in Figure 1 [8]. The magnetic fluid acts as both a valve and a piston. A magnetic fluid valve, held in place by a stationary magnet placed below a pump plate, is always present in the short section of channel between the inlet and outlet of a pumping loop. A magnetic fluid plug, serving as a piston, is drawn around the loop by a rotating magnet placed above the pump plate. As the mobile plug is drawn around the loop, water is drawn into the loop through the inlet, and forced out through the outlet. If the mobile plug reaches the valve plug, it becomes the valve plug. A new mobile plug (piston plug) is generated from the valve plug by the rotating magnet. The maximum flow rate achieved was $45.8 \mu \mathrm{l} / \mathrm{min}$ and the maximum pump head achieved was $135 \mathrm{~mm}$ water $(1320 \mathrm{~Pa})$. However, the pump could not get rid of the disadvantage caused by channel contamination from the magnetic fluid and effluence of the magnetic fluid.
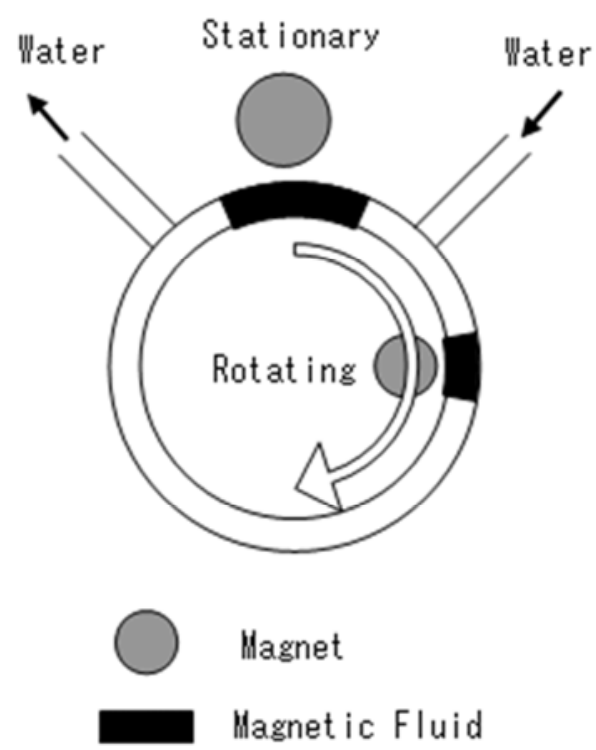

Figure 1. Hatch et al.'s pump.

Recently, Fu et al. performed experiments on micropumps based on nearly the same principle as for Hatch et al.'s pump [9]. However, two magnetic fluid plugs always rotate in a circular loop; one plug works as a piston and the other plug serves as a valve alternately. The maximum flow rate achieved was $128 \mu \mathrm{l} / \mathrm{min}$ for zero pump head; however, the flow rate was not measured for higher pump heads.
Kim et al. proposed a peristaltic micropump using magnetic fluid shown in Figure 2 [10]. Diaphragm between upper and lower channels is made of silicon rubber. Collected magnetic fluid lump in the lower loop channel, drawn by a rotating magnet placed above a pump plate, deforms the silicon rubber diaphragm and then the deformed diaphragm pushes and pumps water in the upper U-shaped channel. The maximum flow rate was $3.8 \mu \mathrm{l} / \mathrm{min}$; however, the maximum pump head is not described in their paper (but is presumed to be nearly zero).

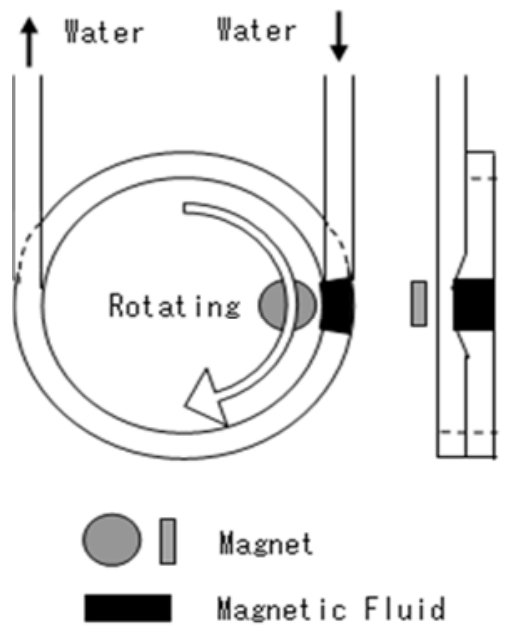

Figure 2. Kim et al. 's pump.

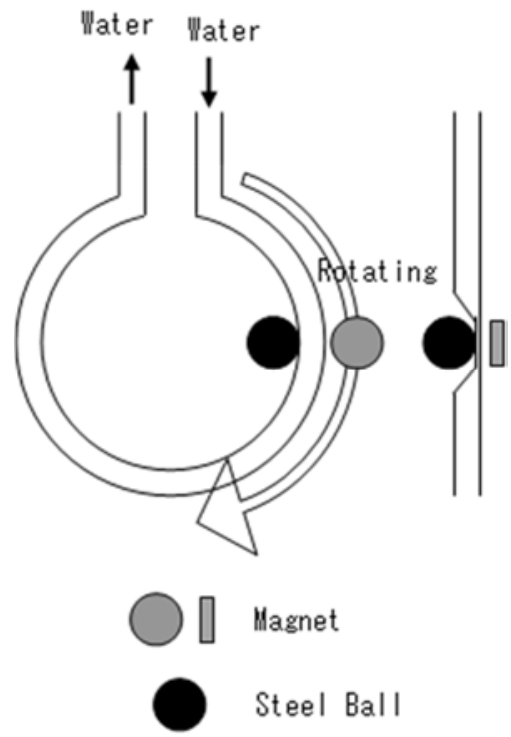

Figure 3. Du et al. 's pump.

Du et al. proposed a peristaltic micropump using magnetic material balls (steel balls) shown in Figure 3 [11]. Steel balls on a polydimethylsiloxane (PDMS) channel are attracted by rotating magnets placed below a PDMS pump plate. The peristaltic deformation of the PDMS membrane pushes and pumps fluid in the channel. The maximum flow rate obtained was $490 \mu \mathrm{l} / \mathrm{min}$ and the maximum pump head achieved was $60.4 \mathrm{~mm}(592 \mathrm{~Pa})$. However, there remain problems in this pump, endurance of deforming PDMS membrane and 
relatively large sizes ( 3 to $5 \mathrm{~mm}$ ) of the steel balls which rotate outside the pump plate.

In this study, first, is tested a micropump using rotational motion of magnetic material balls, based on basically the same principle as for Hatch et al.'s pump. However, the magnetic fluid is replaced by magnetic and non-magnetic material balls which are in a line alternatively, in order to dissolve the defects of Hatch et al.'s pump. Secondly, is developed a micropump using reciprocating motion of magnetic material balls, in order to improve the pump performance. The both micropumps can be incorporated into microfluidic devices (tips) and can pump arbitrary kind of liquid.

\section{Rotational Motion Pump}

\subsection{Operating Principle}

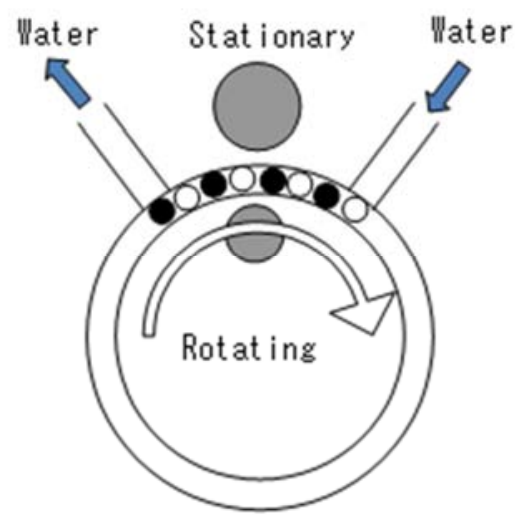

(a)

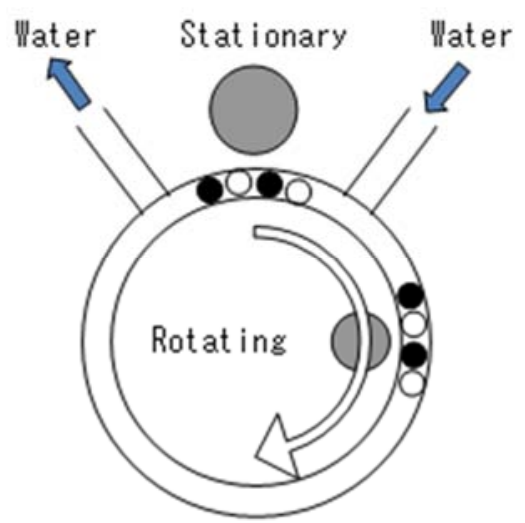

(b)
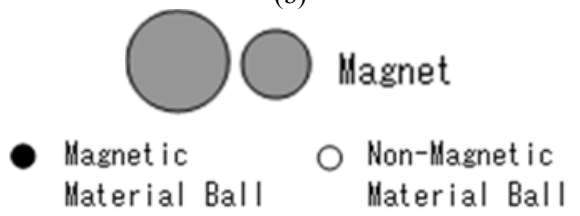

Figure 4. Operating principle of rotational pump.

Figure 4 explains the operating principle of the present micropump based on rotational motion of magnetic material balls. In a closed-loop channel, magnetic and non-magnetic material balls are in a line alternatively (Figure 4(a)). The balls act as both a valve and a piston. A valve part, held in place by a stationary magnet, is always present in the short section of channel between the inlet and outlet of the pumping loop (Figure 4(b)). A piston part is drawn around the channel by an external rotating magnet. When the piston part is drawn around the pumping loop, fluid (such as water) is drawn into pumping loop through the inlet, and forced out through the outlet. If the piston part reaches the region of the stationary magnet, it becomes the valve part again (Figure 4(a)). A new piston part is generated and is drawn by the rotating magnet (Figure 4(b)).

\subsection{Experiments}

Experiments were performed on two pumps with smaller and larger channel cross-sections. Hereafter, these pumps are respectively referred to a "smaller pump" and a "larger pump".

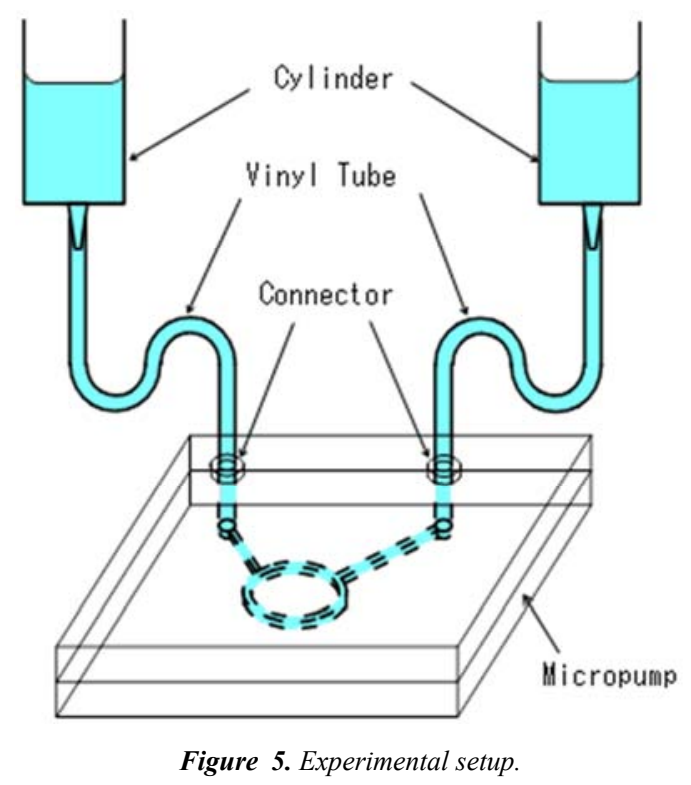

In the smaller pump, the diameter of the pumping loop is $10.5 \mathrm{~mm}$. The circular cross-section of the pumping loop with $1 \mathrm{~mm}$ inner diameter is realized by machinery cutting on upper and lower acryl plates by using a ball end-mill. Five stainless steel (Japanese Industrial Standards (JIS) SUS-440C, magnetic material) balls of $0.8 \mathrm{~mm}$ in diameter and five alumina $\left(\mathrm{Al}_{2} \mathrm{O}_{3}\right.$, non-magnetic material) balls of $0.9 \mathrm{~mm}$ in diameter are in a line alternatively inside the pumping loop. By this arrangement, the balls do not make a cluster (chain) even if the magnetic material balls are magnetized in a magnetic field. The diameters of $0.8 \mathrm{~mm}$ and $0.9 \mathrm{~mm}$ for stainless steel balls and alumina balls, respectively, were selected by the reason that they are commercially available.

In the larger pump, the diameter of the pumping loop is $14.3 \mathrm{~mm}$. The inside diameter of the pumping loop with a circular cross-section is $2.3 \mathrm{~mm}$. Four SUS-440C balls of 2.0 $\mathrm{mm}$ in diameter and four alumina balls of $2.03 \mathrm{~mm}$ in diameter are in a line alternatively inside the pumping loop.

The upper and lower plates are attached by burning in an 
electric furnace at $140^{\circ} \mathrm{C}$ during 2 hours. After this attaching, the pumping loop with circular cross-section is formed between the upper and lower plates.

Figure 5 shows the experimental setup of the present micropumps. The pumped fluid is water in the present experiments. Two cylindrical containers are connected to the inlet and outlet of the pump in order to measure the flow rate and pump head. Before each experiment, water is filled up to the same levels in the two containers. After the start of experiments, the water levels in the two containers are measured in order to obtain the flow rate and pump head from the difference in the liquid levels.

Both the stationary and revolving magnets are a neodymium circular-plate magnet with a diameter of $10 \mathrm{~mm}$, a thickness of $5 \mathrm{~mm}$ and a surface magnetic flux density of $350 \mathrm{mT}$. The stationary magnet is placed on the upper surface of the upper pump plate. The revolving magnet is rotated by a motor just below the lower surface of the lower pump plate. The rotational speed is controlled by a gear-head and a controller, and measured by a tachometer.

\subsection{Experimental Results}

From results of preparatory experiments, it was decided that five magnetic and five non-magnetic material balls are put in the pumping loop for the smaller pump, and four magnetic and four non-magnetic material balls are inserted in the pumping channel for the larger pump. The rotational speed of the revolving magnet is set to $48 \mathrm{rpm}$ for both the pumps. The magnetic material balls cannot follow the magnet in higher speeds than this rotational speed. During the pumping operation, six balls and four balls are worked as the valve part and the piston part, respectively, in the smaller pump, and four balls and four balls are worked as the valve part and the piston part, respectively, in the larger pump.

Before the experiments, water is filled up to the same levels in the two containers. After the start of experiments, the water levels in the two containers are measured until the difference in the liquid levels does not change. The pumped water and the pump head are calculated from the changes in the liquid levels and the difference in the liquid levels, respectively. The flow rate is calculated from the increase in the pumped water.

Figure 6 shows the relation between the flow rate and the pump head, for both the larger and smaller pumps, obtained from the experiments. The maximum errors included in the experimental data are estimated to be $\pm 0.09 \mathrm{~mL} / \mathrm{min}$ in the flow rate and $\pm 0.5 \mathrm{~mm}$ in the pump head. In the case of larger pump, the flow rate does not decrease monotonously with increasing pump head. The cause for this is attributable to pump operating principle itself, i.e. the magnetic balls cannot follow completely the rotation of the external rotating magnet for relatively high flow rate and high pump head. The maximum flow rate obtained for the larger pump, $\sim 2 \mathrm{~mL} / \mathrm{min}$, is large enough as a micropump. However, the maximum pump head, $\sim 15 \mathrm{~mm}$, is small even for a micropump. The cause for the small pump head is water leakage through the gap between the balls and the wall in the pumping channel. It is desirable to increase the pump head furthermore.

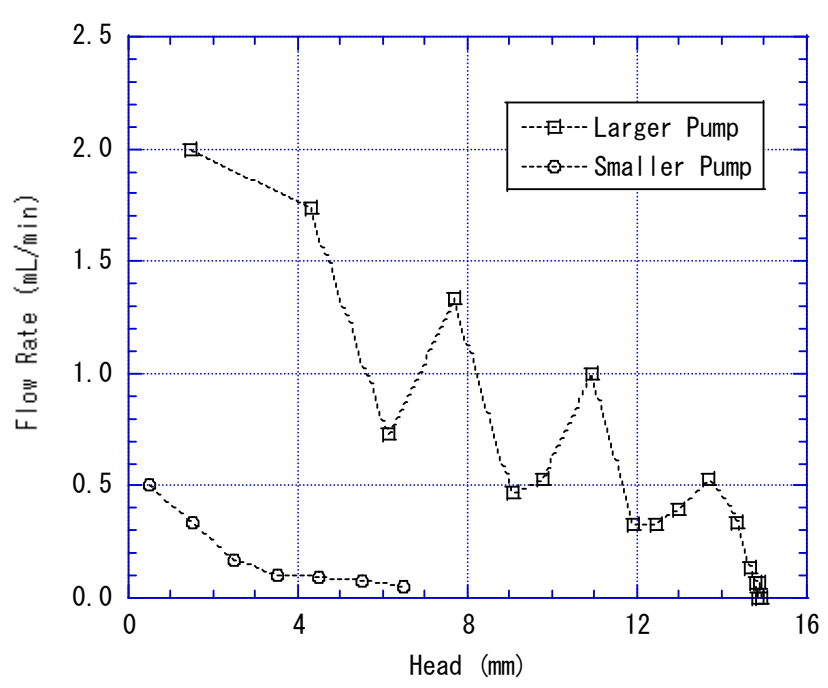

Figure 6. Pump performance for rotational pump.

\section{Reciprocating Motion Pump}

\subsection{Operating Principle}

Figure 7 shows the operating principle of the present micropump based on reciprocating motion of magnetic material balls. The micropump combines reciprocating motion of magnetic material balls and four passive check valves (including one non-magnetic material ball in each valve). By using four passive check valves, the micropump can send water from the inlet to the outlet throughout the reciprocating motion (i.e. during both forward and backward motions) of the magnetic material balls. Figures 8(a) and (b) show the plan view and front view of the passive check valve, respectively. Figure 8(c) presents the bird's-eye view where the non-magnetic material ball is not illustrated in order to make the flow channel visible easily. The right-hand side of the valve space with a ball is connected to a flow channel with a circular cross-section, though the left-hand side is connected to a flow channel with a rectangular cross-section.

As shown in Figure 7(a), when the magnetic material balls, drawn by an external magnet, move towards this side in the pumping channel, water is pumped from the inlet to the outlet, through the check valve on the upper right, the pumping channel and the check valve on the lower left. The check valves on the lower right and the upper left are nearly closed during this process. On the other hand, as shown in Figure 7(b), when the magnetic material balls move towards the opposite side in the pumping channel, water is pumped from the inlet to the outlet, through the check valve on the lower right, the pumping channel and the check valve on the upper left. The check valves on the upper right and the lower left are nearly closed during this process. By these two processes, the micropump can send water from the inlet to the outlet throughout the reciprocating motion (i.e. during both forward and backward motions) of the magnetic material balls. 


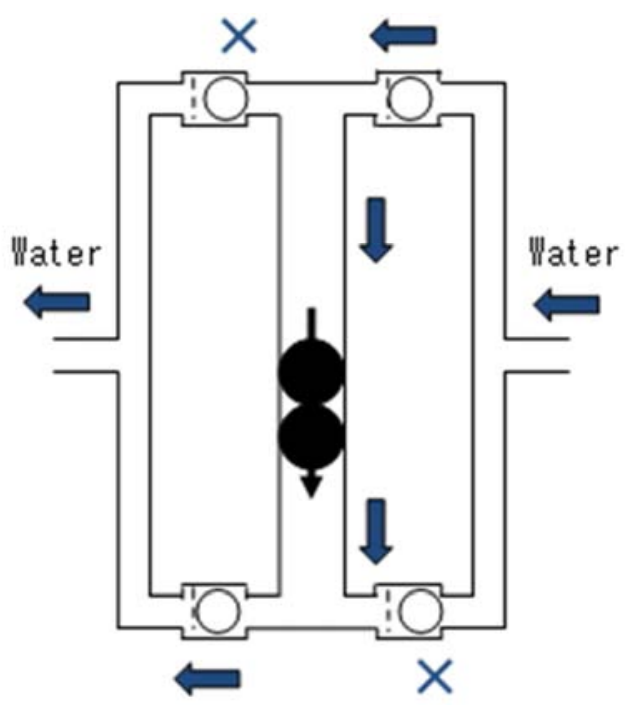

(a)

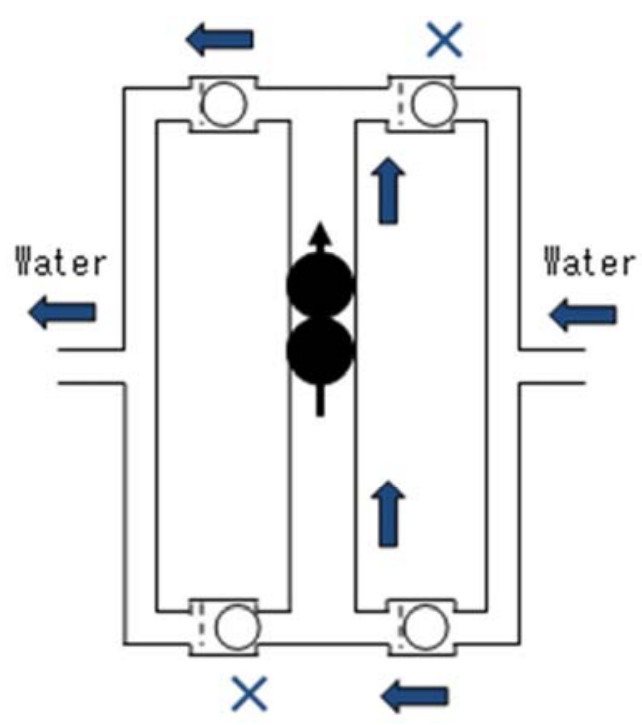

(b)

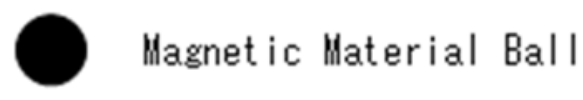

\section{Non-llagnetic Material Ball}

Figure 7. Operating principle of reciprocating pump.

\subsection{Experiments}

The pumping channel having a length of $34 \mathrm{~mm}$ and an inner diameter of $2.00 \mathrm{~mm}$ or $2.05 \mathrm{~mm}$ is made of PTFE. The magnetic material ball is a SUS-440C ball with an outer diameter of $1.98 \mathrm{~mm}$ or $2.00 \mathrm{~mm}$. The magnetic material balls are put into reciprocating motion by an external neodymium magnet, with a diameter of $10 \mathrm{~mm}$, a thickness of $5 \mathrm{~mm}$ and a surface magnetic flux density of $350 \mathrm{mT}$. The magnet performs reciprocating motion by a motor for a toy, using two $1.5 \mathrm{~V}$ batteries, and a slider device. The frequency of the reciprocating motion is changed from $\sim 1 \mathrm{~Hz}$ to $\sim 5 \mathrm{~Hz}$.

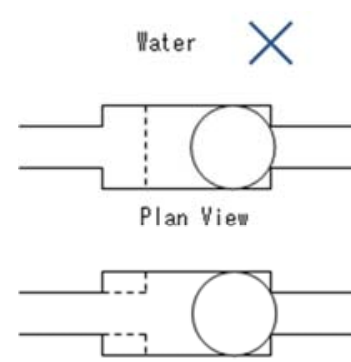

Front View

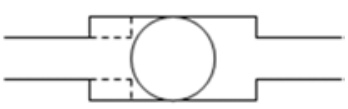

Front View

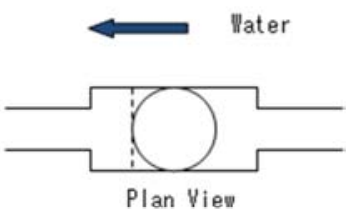

(a)

(b)

Non-Magnet ic

Haterial Ball

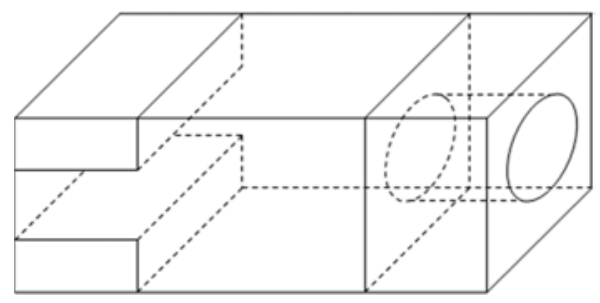

(c)

Figure 8. Passive check valve.

The passive check valve includes one alumina $\left(\mathrm{Al}_{2} \mathrm{O}_{3}\right)$ ball, with an outer diameter of $1.5 \mathrm{~mm}$, in fluid space with $1.7 \mathrm{~mm}$ $\mathrm{x} 1.7 \mathrm{~mm}$ in cross-section and $2.5 \mathrm{~mm}$ in length (See Figure $8(\mathrm{c})$ ). When the ball moves to one side direction by fluid force, the ball plugs the outlet to the connecting channel with a circular cross-section (See Figure 8(a)). On the other hand, when the ball moves to the other side direction, the ball does not plug the outlet to the connecting channel with a rectangular cross-section (See Figure 8(b)). Thus, the valve works as a passive check valve.

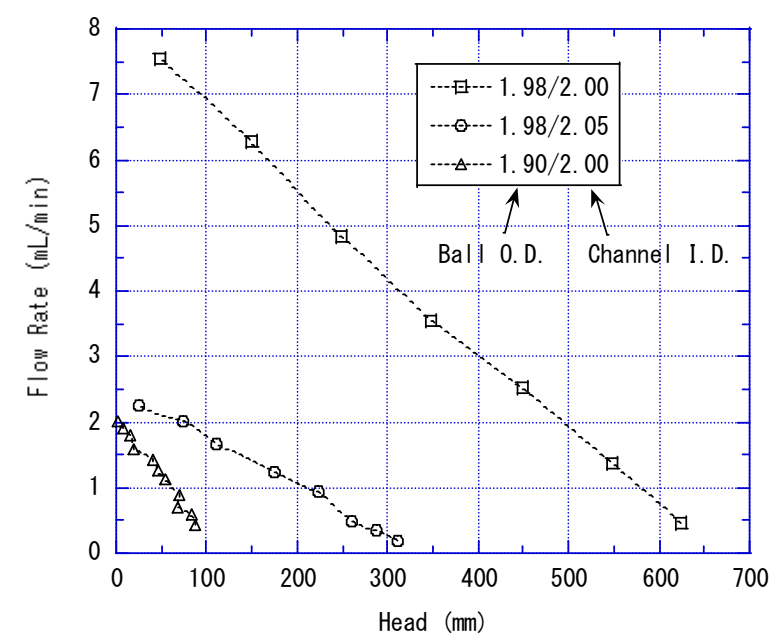

Figure 9. Pump performance for reciprocating pump.

The flow channels, including the pumping channel and the valve channels, are fabricated on upper and lower acryl plates by machinery cutting with an end-mill. The upper and lower 
acryl plates, including the PTFE tube in the pumping channel, are attached by burning in an electric furnace at $110^{\circ} \mathrm{C}$ during 5 hours.

The experimental setup and the experiment procedure are the same as for the micropump using rotational motion of magnetic material balls, explained in Sec. 3.2.

\subsection{Experimental Results}

The reciprocating frequency was set at $1.24 \mathrm{~Hz}, 2.43 \mathrm{~Hz}$, $4.05 \mathrm{~Hz}$ and $4.77 \mathrm{~Hz}$. The best pump performance was obtained at $4.05 \mathrm{~Hz}$ in all the three combinations of the ball outer diameter and the PTFE tube inner diameter. Figure 9 shows the relation between the flow rate and the pump head for the case of reciprocating frequency of $4.05 \mathrm{~Hz}$. The maximum errors included in the experimental data are estimated to be $\pm 0.18 \mathrm{~mL} / \mathrm{min}$ in the flow rate and $\pm 1 \mathrm{~mm}$ in the pump head. The relation between the flow rate and the pump head is expressed nearly a straight line, for three combinations of the ball outer diameter and the PTFE tube inner diameter. The smaller the gap between the ball outer diameter and the channel inner diameter becomes, the better the pump performance becomes. The maximum flow rate and the maximum pump head are $\sim 7.5 \mathrm{~mL} / \mathrm{min}$ and $\sim 625 \mathrm{~mm}$, respectively, for the combination of the ball outer diameter of $1.98 \mathrm{~mm}$ and the PTFE tube inner diameter of $2.00 \mathrm{~mm}$. These values are sufficient as a micropump. Also, the present micropump can be incorporated into microfluidic devices (tips) and can pump arbitrary kind of liquid.

\section{Conclusion}

Micropumps using rotational and reciprocating motions of magnetic material balls were proposed and studied experimentally. Both the micropumps can be incorporated into microfluidic devices (tips) and can pump arbitrary kind of liquid.

For the rotational motion micropump, the maximum flow rate obtained and the maximum pump head achieved are $\sim 2$ $\mathrm{mL} / \mathrm{min}$ and $\sim 15 \mathrm{~mm}$, respectively. Though the maximum flow rate is large enough as a micropump, the maximum pump head is small even for a micropump. It is desirable to increase the pump head furthermore. For the reciprocating motion micropump, the maximum flow rate obtained and the maximum pump head achieved are $\sim 7.5 \mathrm{~mL} / \mathrm{min}$ and $\sim 625$ $\mathrm{mm}$, respectively. These values of the pump performance are sufficient as a micropump.

\section{Acknowledgements}

A part of this work was supported by JSPS (The Japan Society for the Promotion of Science) KAKENHI Grant Number 24656130.

\section{References}

[1] C. Zhou, H. Zhang, Z. Li, and W. Wang, "Chemistry pumps: a review of chemically powered micropumps," Lab on a Chip, vol. 16, pp. 1797-1811, 2016.

[2] F. R. Munas, Y. W. R. Amarasinghe, and D. Dao, "Review on MEMS based micropumps for biomedical applications," IJIRSET, vol. 4, issue 7, pp. 5602-5615, 2015.

[3] S. Yokota, "A review on micropumps from the viewpoint of volumetric power density," Bulletin of the JSME, Mech. Eng. Reviews, vol. 1, no. 2, pp. 1-11, 2014.

[4] F. Abhari, H. Jaafar, and N. A. Yunus, "A comprehensive study of micropumps technologies," Int. J. Electrochem. Sci., vol. 7, pp. 9765-9780, 2012.

[5] A. K. Au, H. Lai, B. R. Utela, and A. Folch, "Microvalves and micropumps for bio MEMS," Micromechanics, vol. 2, pp. 179-220, 2011.

[6] Editing Committee of Handbook of Micro- and Nano- Heat and Fluid, "Handbook of Micro- and Nano- Heat and Fluid," NTS Inc., Tokyo, Japan, 2006, pp. 378-397 [In Japanese].

[7] I. Yanagisawa, "Electroosmotic flow pump mountable on chip," Electric Parts and Materials, vol. 44, no. 11, pp. 45-48, 2005 [In Japanese].

[8] A. Hatch, A. E. Kamholz, G. Holman, P. Yager, and K. F. Bohringer, "A ferrofluidic magnetic micropump," J. of Microelectromechanical Systems, vol. 10, no. 2, pp. 215-221, 2001.

[9] L. M. Fu, W. C. Fang, T. F. Hong, and C. Y. Lee, "A magnetic micropump based on ferrofluidic actuation," Int. J. of Automation and Smart Technology, vol. 4, no. 2, pp. 77-82, 2014.

[10] E. G. Kim, J.-G. Oh, and B. Choi, "A study on the development of a continuous peristaltic micropump using magnetic fluids," Sensors and Actuators A, vol. 128, pp. 43$51,2006$.

[11] M. Du, X. Ye, K. Wu, and Z. Zhou, "A peristaltic micro pump driven by a rotating motor with magnetically attracted steel balls," Sensors, vol. 9, pp. 2611-2620, 2009. 